\title{
Compositeurs au pouvoir en France sous la IIIème République
}

\section{Myriam Chimènes}

On connaît le rôle du pouvoir monarchique en France sur le développement des arts qu'il favorise au prix d'un sévère contrôle. C'est ce qui autorise les seuls artistes désignés à exercer une véritable hégémonie, dont, en musique, Lully est le représentant le plus marquant sous le règne de Louis XIV.1 Quant à la politique artistique du pouvoir républicain, elle a été étudiée dans le domaine des arts plastiques, mais demeurait encore relativement inconnue dans le domaine musical (sauf pour la période contemporaine) jusqu'à notre étude récente sur "Le budget de la musique sous la IIIeme République"."

La IIIeme Republique (1870-1940) présente un terrain d'investigations privilégié, parce qu'elle constitue une période constitutionnelle stable et surtout parce qu'elle marque l'avènement définitif du pouvoir républicain, la liquidation de la Révolution Française, après un siècle prèsque de balbutiements et de tentatives laborieuses de mise en place et de stabilisation de la démocracie. ${ }^{3}$

Les études consacrées aux arts plastiques mettent en évidence l'existence d'un "art officiel" dont la postérité est bien en deçà de celle réservée à l'art marginalisé par le pouvoir de l'époque. ${ }^{4}$ Ainsi, au regard de l'histoire, les impressionistes ont longtemps été considérés comme seuls à caractériser leur temps, leur génie repoussant dans l'ombre (juste revers des choses) les représentants de l'académisme. A contre courant de ces raccourcis historiques, le Musée d'Orsay illustre une nouvelle réflexion sur l'histoire de l'art, soucieuse de relativiser et de permettre d'appréhender de manière plus juste la production artistique de l'époque. Le travail de l'historien ne peut pas en effet se limiter à la formulation de jugements esthétiques, mais il doit aussi tenter de reconstituer le tissu culturel et artistique d'une époque, dans un but purement objectif et descriptif.

En accordant pendant longtemps une plus grande place à la marginalité, l'histoire, tout en reconnaissant et célébrant le génie des "refusés", rendait en même temps justice aux novateurs relégués alors au ban de la société artistique officielle. Dans le domaine de la musique, la situation est analogue, à ceci près qu'un utopique "Musée d'Orsay de la musique" devrait équilibrer le répertoire des concerts afin de confronter les diverses tendances esthétiques qui ont coexisté. On comprend aisément que le parallèle entre arts plastiques et musique devient absurde, la valeur symbolique, les modes de représentation et de diffusion, et le "marché" se révèlant à l'évidence fondamentalement différents.

On ne peut cependant qu'insister sur ce point commun fondamental: en musique comme en peinture, sous la IIIème République, la marginalité n'a jamais reçu le soutien de l'Etat. Par définition, les novateurs ne furent jamais 
acceptés par les institutions officielles. Ceux que retient la mémoire collective (souvent à l'exclusion de tous les autres) sont précisément ceux que la mémoire officielle entendait délibérément évacuer. Au regard de l'histoire, les grands sont donc les refusés, les maudits. Ce chassé croisé entre mémoire collective et mémoire officielle nous paraît éminemment symptomatique.

Courbet ou Manet furent contraints d'exposer en dehors des salons officiels, Corot fut refusé au Prix de Rome. L'histoire de la musique présente des exemples analogues qui mettent en lumière le fossé qui sépare les représentants de l'art académique, véritables "musiciens officiels" des esprits indépendants et novateurs, spontanément libérés de toute contrainte. Ainsi, Debussy passa près de dix années à la recherche d'un théâtre pour montrer Pelléas et Mélisande et Ravel, en 1905, ne fut pas même admis à concourir pour le Prix de Rome. Ces créateurs étaient-ils à ce point considérés comme dangereux et subversifs?

Néanmoins, quelques exceptions confirment la règle. Debussy obtint le prix de Rome en "trompant l'ennemi”, c'est à dire en se pliant stratégiquement aux rẻgles académiques. Alfred Bruneau n'était pas dupe, qui dénonçait quelques années plus tard le parfait simulateur: "L'Institut, sans méfiance, l'envoya, le diplôme de bon et studieux élève en poche, à la Villa Médicis. On cherchera vainement d'ailleurs dans l'académique cantate de L'Enfant prodigue, dont le doux Guiraud, son maître, était si fier, la trace des tendances qui, à présent, ravissent les premiers et scandalisent les autres". Les représentants de l'art académique se sentaient probablement menacés, ce qui explique des positions peut-être plus défensives que réellement motivées par la volonté de dicter une esthétique. Le Musée d'Orsay a rétabli l'equilibre, mis les oeuvres en perspective en exposant, outre les peintres impressionnistes, les "pompiers", c'est à dire les représentants de l'art officiel, afin de dresser un panorama complet de l'histoire de l'art de cette époque, et de montrer comment les petits maîtres peuvent aider à comprendre les grands. On doit pouvoir expliquer de manière comparable sinon strictement analogue le terrain musical de la IIIème République, afin de replacer dans son contexte musical la célèbre "trilogie" Fauré - Debussy - Ravel (parmi eux, Fauré est le seul à occuper des fonctions officielles), qui véhicule schématiquement presqu'à elle seule l'image musicale de cette époque. S'ils en sont les plus illustres représentants, ils n'en ont neanmoins pas l'exclusivité. Il convient aussi d'étudier les réactions du public, "il faut vérifier si les choix de la postérité recouvrent ou non ceux des contemporains et pourquoi".

Il est donc classique d'opposer les "pompiers" aux impressionnistes, le salon officiel à celui des refusés. Les termes mêmes sont éloquents. La situation est-elle comparable en musique? Y avait-il des "pompiers" de la musique? Qui détenait le pouvoir musical en France? Comment s'exerçait-il?

En matière artistique, la IIlème République délègue ses pouvoirs à l'Académie des Beaux-Arts. Celle-ci a le pouvoir exclusif d'organiser et de sanctionner à la fois lenseignement. Le prix de Rome constitue le seul filtre capable de retenir les artistes doués. Cet écrémage garantit la qualité, le respect 
des normes esthétiques et permet ensuite d'alimenter le répertoire contemporain de l'Opéra, institution officielle par excellence. La section de composition musicale gère donc le prix de Rome: elle donne les sujets, rédige les programmes et juge les résultats avant de les soumettre au Ministre. Elle contrôle et sanctionne ensuite les travaux des pensionnaires pendant leur séjour à la Villa Médicis. Pour les arts plastiques, l'Académie contrôle l'enseignement des Beaux-Arts et décide des admissions aux salons annuels. En ce qui concerne la musique, elle exerce un monopole indirect sur les créations à l'Opéra, le cahier des charges de ce théâtre stipulant l'obligation de donner tous les deux ans un ouvrage d'un compositeur français recruté parmi les anciens lauréats du prix de Rome. Ces réflexions préliminaires justifient l'intérêt de brosser un portrait des compositeurs qui détiennent ces véritables postes de commandes que sont les fauteuils à l'Institut.

L'Académie des Beaux-Arts comprend cinq sections: peinture (12 membres), sculpture ( 7 membres), architecture ( 8 membres), gravure (4 membres) et composition musicale ( 6 membres)(7). Ainsi, 27 musiciens siègent à l'Académie des Beaux-Arts au cours de la IIlème République. Schématiquement, trois critères principaux semblent autoriser l'accession d'un musicien au fauteuil d'académicien: la carrière d'enseignant au Conservatoire, la réussite dans le domaine de l'art lyrique et la notoriété acquise comme chef d'orchestre ou comme virtuose. Nous choisirons ici les exemples les plus marquants.

\section{L'enseignement au Conservatoire}

La responsabilité d'une classe de composition au Conservatoire est une voie d'accès efficace vers l'Academie. Victor Massé, Henry Reber, François Bazin, Charles Lenepveu, Ernest Guiraud, Théodore Dubois, Charles- Marie Widor, Gabriel Fauré et Paul Dukas en témoignent. C'est ce qui explique que les candidats qui concourent pour le Prix de Rome sont quasiment toujours les eleves de certains membres du jury. D'ailleurs, les palmarès des concours de Rome sont éloquents, car ils permettent de constater l'influence notable des jurés qui cumulent les deux fonctions. Charles Lenepveu présente sans doute l'exemple le plus significatif. Il siège à L'Academie durant près de quinze ans - il y est élu deux ans après sa nomination comme professeur de composition au Conservatoire. Pendant son règne, 27 de ses éleves seront couronnés, dont 10 seront les lauréats du premier Grand Prix de Rome. Sa virtuosité en la matière reste inégalée.

Ernest Guiraud (1837-1892), dont le père était lui- même lauréat du Premier Grand Prix de Rome, remporte à son tour cette distinction en 1859. Il débute comme timbalier à l'Opéra Comique. Son ballet Gretna Green est créé à l'Opéra en 1873 et son opéra Le Piccolino à l'Opéra Comique en 1876. Il enseigne l'harmonie au Conservatoire de 1876 à 1880 , puis la 
composition jusqu'a sa mort. Il est membre du jury du Concours de la Ville de Paris en 1887-88 et fait partie de la Commission des Auditions musicales pour l'Exposition Universelle de 1889 au cours de laquelle on joue une de ses oeuvres. Il est élu à l'Académie en 1891. C'est sa troisième candidature, après s'être vu préférer Saint-Saens puis Léo Delibes. Il n'a plus qu'un an à vivre. Son influence y sera donc limitée.

Eleve d'Ambroise Thomas, Théodore Dubois (1837-1924), qui est plus connu aujourd'hui comme auter d'un célèbre traité d'harmonie, remporte le Premier Grand Prix de Rome en 1861. Organiste, il est maître de chapelle à Sainte-Clotilde puis à la Madeleine. Il enseigne l'harmonie au Conservatoire de 1871 à 1891, puis la composition jusqu'en 1896. Il remporte le Premier Prix au Concours musical de la Ville de Paris en 1878. Son ballet La Farandole est monté à l'Opéra en 1883. Il est élu à l'Académie en 1894, ce qui favorise sa nomination à la direction du Conservatoire deux ans plus tard. Le "scandale Ravel" (cf infra) le contraindra à démissionner de ce poste en 1905.

Elevve d'Ambroise Thomas, Charles Lenepveu (1840-1910) remporte le Premier Grand Prix de Rome en 1865. Il est membre de la Société Nationale dès 1871. Son opéra Le Florentin est créé à l'Opéra Comique en 1874. Il est professeur d'harmonie au Conservatoire de 1880 à 1894, puis de composition jusqu'à sa mort. Il fait partie de la commission des Auditions musicales de l'Exposition Universelle de 1889 au cours de laquelle une de ses oeuvres est jouée. Il est élu à sa première candidature à l'Académie en 1896. En 1905, l'année où Ravel n'est pas même admis à concourir pour le Prix de Rome, les cinq lauréats sont ses éleves. ${ }^{8}$

Charles-Marie Widor (1844-1937) est d'une famille d'organistes et de facteurs d'orgues. Elève de Fétis à Bruxelles, il ne fait pas ses études au Conservatoire et ne concourt pas pour le Prix de Rome. Il est organiste à Saint-Sulpice de 1870 à 1934. Son opéra Mâ̂tre Ambros est créé à l'Opéra Comique en 1886. Une de ses oeuvres est jouée à l'Exposition Universelle de 1889. Il succède à Franck comme professeur d'orgue au Conservatoire en 1890, puis à Dubois à la classe de composition en 1896. Il fait partie de la Commission des Auditions musicales de l'Exposition Universelle de 1900. Il est élu à l'Académie où il pose sa candidature pour la troisième fois en 1910. Il en sera le secrétaire perpétuel de 1914 jusqu'à sa mort.

Gabriel Fauré (1845-1924) est l'élève de Saint-Saëns à l'Ecole Niedermeyer. Il présente plusieurs points communs avec Widor: il ne fait pas ses études au Conservatoire, il ne se présente pas au Prix de Rome et il est organiste (à Rennes de 1865 à 1870, puis à Paris, successivement à Saint-Honoré d'Eylau, à Saint-Sulpice et à la Madeleine). Il enseigne à l'Ecole Niedermeyer. A deux reprises, en 1885 et en 1893, il obtient le Prix Chartier (un prix de l'Institut). Il fait plusiers fois partie du jury du Concours de la Ville de Paris et du Concours Cressent. Il devient inspecteur de l'enseignement musical en 1892. Il est nommé professeur de composition au Conservatoire en 1896. Il fait partie de la Commission des Auditions musicales de l'Exposition Universelle de 1900. En 1905, il est nommé Directeur du Conservatoire, à la suite de la démission 
de Théodore Dubois. Il sera élu à l'Académie en 1909.

Eléve de Guiraud, Paul Dukas (1865-1935) obtient le Second Grand Prix de Rome en 1888. Son opéra Ariane et Barbe Bleue est créé à l'Opéra Comique en 1907. Nommé à la classe d'orchestre au Conservatoire en 1910, il en démissionne en 1913. Il est professeur de composition à l'Ecole normale de musique, membre du Conseil supérieur du Conservatoire et inspecteur général de la musique. Il succède à Widor à la classe de composition au Conservatoire en 1928. Il pose pour la première fois sa candidature à l'Académie où il est élu en 1934. Il mourra l'année suivante.

\section{Le theâtre lyrique}

"L'Opéra est une sorte d'institution nationale, qui intéresse davantage l'histoire de l'art officiel que de l'art vivant" "Cette réalité, que confirment les documents financiers ('Oopéra absorbe alors environ $80 \%$ du budget consacré à la musique), montre bien que l'Opéra est pendant longtemps considéré comme le seul moyen d'expression de lart musical, tout entier confiné dans sa fonction théâtrale. Dans ce domaine encore, l'Académie est toute puissante. Le cahier des charges de l'Opéra impose la création d' "actes nouveaux" de compositeurs français, obligatoirement recrutés parmi les anciens élèves de Rome. C'est ce qui explique que la lecture de la liste des oeuvres créées à l'Opéra est essentiellement une longue litanie de noms obscurs, lauréats du Prix de Rome, qui n'ont souvent pour autre point commun que leur absence totale de notoriété et leur quasi-totale disparition de la mémoire collective. Néanmoins, en dehors des prix de Rome, quelques noms se détachent, de compositeurs qui recueillent les suffrages du public. Le maintien, voire le retour à l'affiche, est indéniablement preuve de réussite et incite la direction à̆ programmer de nouvelles oeuvres du compositeur à succès. En effet, si la subvention de l'opéra est importante, les recettes restent cependant indispensables. Il y a bien évidemment relation de cause à effet: qui dit succés, dit augmentation des recettes. La reconnaissance du public est donc prometteuse en ce domaine: elle peut conduire un compositeur vers l'Institut. Il n'est alors pas indispensable qu'elle s'accompagne de preuves dans le domaine de l'enseignement. Les musiciens qui doivent leur fauteuil d'académicien au fait de s'être brillamment illustrés dans le domaine de l'opéra sont Ambroise Thomas, Charles Gounod, Léo Delibes, Emile Paladilhe, Alfred Bruneau, Georges Hüe, Jules Massenet et Gustave Charpentier.

Ambroise Thomas (1811-1896) est issu d'une famille de musiciens. II obtient le Prix de Rome en 1832. Il compose de manière industrielle pour la scène et doit son élection à l'Académie en 1851 aux succès que viennent de remporter à l'Opéra Comique Le Caïd (1849) et Le Songe d'une nuit d'été (1850). Il sera professeur de composition au Conservatoire de 1856 à 1871, puis directeur jusqu'à sa mort. Il est l'auteur de 25 oeuvres lyriques parmi lesquelles Mignon constitue l'un des plus grands succès de l'histoire de l'opéra 
français. Il siège à l'Académie pendant 45 ans.

Charles Gounod (1818-1893) obtient le Prix de Rome en 1839. Il ne compose pas moins de dix opéras avant son élection à l'Académie en 1866. En 1859, Faust lui a valu la reconnaissance du grand public.

Organiste, Léo Delibes (1836-1891) compose de nombreuses opérettes avant de devenir chef de choeurs à l'Opéra. Ses ballets Coppélia (1870) et Sylvia (1876) le rendent populaire, et son opéra Lakmé (1883) obtient un triomphe. Professeur de composition au Conservatoire depuis 1881, il est élu à sa seconde candidature à l'Académie en 1884.

Eleve d'Ambroise Thomas, Jules Massenet (1842-1912) obtient le Prix de Rome en 1863. Créé à l'Opéra en 1877, son opéra Le Roi de Lahore tient l'affiche pour 30 représentations et lui vaut une réputation internationale. Il est nommé professeur de composition au Conservatoire en 1878 (il démissionnera en 1896) et élu la même année à l'Académie où il se présente pour la première fois. Il a alors 36 ans et il devance Saint-Saëns. Extrêmement populaire auprès du public, il est méprisé par ses confrères qui lui pardonnent mal ses capacités de séduction.

Elève chéri de Massenet, Gustave Charpentier (1860-1956) obtient le Prix de Rome en 1887. Sa carrière, atypique, est marquée par le triomphe de Louise à l'Opéra Comique en 1900. C'est sur la sollicitation des membres de l'Académie qu'il fait acte de candidature et est élu en 1912.

\section{La réputation de chef d'orchestre ou de virtuose}

Occuper les fonctions de chef d'orchestre a l'Opéra ou à l'Opéra Comique, voire d'une des associations symphoniques constitue incontestablement un bon marchepied vers l'Académie. Pour les compositeurs, ces postes-clé garantissent prèsqu'avec certitude la possibilité d'être joué. La notoriété acquise grâce au succès auprès du public semble également un atout non négligeable pour accéder à un poste officiel. Sensibles à la réputation, les professionnels ne sont pas indifférents aux goûts du public, dont les hommages leur servent de caution. Quelques compositeurs doivent en effet leur célébrité à leurs talents d'interprètes avant d'être reconnus comme créateurs - pour certains d'ailleurs, la postérité n'a retenu que leur carrière de chef d'orchestre au détriment de leur production de compositeur. Il s'agit de Camille Saint-Saëns, André Messager, Gabriel Pierné, Alfred Bachelet, Henry Busser et Henri Rabaud.

Camille Saint-Saëns (1835-1921) est sans doute la meilleure illustration de cette catégorie de musiciens. Après des études au Conservatoire, il essuie deux échecs au Prix de Rome. Il enseigne à l'Ecole Niedermeyer de 1861 à 1865. Il fonde la Société Nationale en 1871. Il est titulaire de l'orgue de Saint Merri puis de celui de la Madeleine. Dès le début des années 1860, il acquiert une formidable réputation de compositeur et de pianiste virtuose et entame une carrière internationale. En 1868 son deuxième Concerto pour piano lui vaut un immense succès. En 1881, il est élu à l'Académie où il pose sa candidature 
pour la seconde fois, ayant été battu par Massenct en 1878.

Ami de Fauré, André Messager (1853-1929) est élève de Saint-Saèns à l'Ecole Niedermeyer. Il est organiste successivement à Saint-Sulpice, à Saint-Paul Saint-Louis et à Saint-Marie des Batignolles. Il est chef d'orchestre aux Folies Bergères en 1877. Il obtient un Prix au Concours de la Ville de Paris en 1881. Il est joué à l'Opéra et á l'Opéra Comique. Il est Directeur de la musique et chef d'orchestre à l'Opéra Comique de 1898 à 1903 et directeur artistique à Covent Garden de 1901 à 1907. Il est chef d'orchestre et co-directeur de l'Opéra de 1907 à 1914 et chef de la Société des Concerts du Conservatoire de 1908 à 1919. Il est Directeur de la musique à l'Opéra comique de 1919 à 1920. Il est élu à l'Académie en 1926 à sa quatrième candidature.

Elève de Massenet et de Franck, Gabriel Pierné (1863-1937) obtient le Prix de Rome en 1882. Il succède à Franck à l'orgue de Sainte-Clotilde. Il remporte un prix au Concours de la Ville de Paris de 1900-1903. Il dirige aux Concerts Colonne dès 1903, et succède à Edouard Colonne en 1910 jusqu'en 1934. Son rôle de chef d'orchestre y sera déterminant pour le développement de la création musicale de son temps. Comme Messager, il doit attendre sa quatrième candidature pour être élu à l'Académie en 1924.

Eleve de Guilmant, Franck, Widor et Guiraud, Henry Busser (1872-1973) obtient le Prix de Rome en 1893. Il est chef d'orchestre au Théâtre du Château d'Eau à partir de 1900, à l'Opéra Comique à partir de 1902, à l'Opéra à partir de 1905. Il est nommé à la classe d'ensemble vocal du Conservatoire en 1904 et devient professeur de composition en 1930. Il est élu à l'Académie en 1938.

Henry Rabaud (1873-1949) obtient le Prix de Rome en 1894. Il est chef d'orchestre à l'Opéra de 1908 à 1918, puis directeur de 1914 à 1918 . Il est élu en 1918 à sa première candidature à l'Académie. En 1922, il succède à Fauré à la Direction du Conservatoire.

Deux compositeurs échappent à cette classification, même s'ils peuvent se rattacher par certains aspects à l'une ou l'autre de ces catégories. Il s'agit d'Ernest Reyer et de Florent Schmitt. Quasiment autodidacte, Ernest Reyer (1823-1903) commence la composition en 1850. Il occupe le poste stratégique de bibliothécaire à l'Opéra, ce qui lui permet d'y faire monter plusieurs de ses oeuvres. Il est élu à l'Académie en 1876. Quant à Florent Schmitt (1870-1958), élève de Dubois, Massenet et Fauré, il obtient le Prix de Rome en 1900. Directeur du Conservatoire de Lyon de 1922 à 1924, critique musical au Temps de 1929 à 1939, il est élu à l'Académie en 1936.

Cette présentation schématique des qualités requises pour accéder au pouvoir, c'est à dire, en ce qui concerne un compositeur, pour siéger à l'Académie des Beaux-Arts, appelle quelques commentaires. Nous n'avons dressé ici qu'un résumé volontairement ellyptique du curriculum vitae des musiciens au moment de leur candidature, en mettant l'accent sur les événements marquants de leur carrière qui justifiaient une reconnaissence officielle. Nous n'avons donc ni décliné le catalogue de leurs oeuvres, ni énuméré les fonctions qu'ils cumulent après leur élection. Les avantages sont nombreux. Outre qu'il 
leur garantit quasi-systématiquement d'avoir une oeuvre montée à l'Opéra, ce poste leur permet d'être joué par les grandes associations symphoniques et la plupart des sociétés de concerts, l'intérêt des organisateurs étant bien évidemment de rentrer dans les bonnes grâces des tout-puissants membres de l'Académie des Beaux-Arts. Ces musiciens bénéficient également d'autres postes honorifiques, tels que la présidence des commissions musicales des Expositions Universelles ou la direction du Conservatoire (seule exception, Fauré, après deux échecs à l'Académie, doit au contraire son élection à son poste de directeur du Conservatoire). Les musiciens académiciens semblent les seuls à avoir une légitimité vis à vis du pouvoir politique, à être reconnus dignes de s'inscrire dans la memoire de l'Etat. L'honneur suprême et ultime sera réservé à Fauré qui aura droit à des obsèques nationales.

Nous avons décrit les divers procédés requis pour réussir ce parcours du combattant (avec les quelques exceptions qui confirment la règle). Néanmoins, il est extrêmement important de rappeler que la volonté de les réunir est primordiale. Le déterminisme en la matière est absolument essentiel: en effet, pour être élu à Ínstitut, il convient de faire acte de candidature. Les postulants doivent donc adresser une lettre de candidature à l'Académie dès qu'une vacance est déclarée. La section musicale procède ensuite au classement des candidats. Enfin, la liste ainsi établie est soumise au vote de l'Académie des Beaux-Arts en séance plénière. Les membres plasticiens étant largement majoritaires, on comprend alors aisément la nécessité de s'assurer leurs voix. On peut ainsi mesurer l'influence qu'exercent sur eux leurs collègues musiciens, contrecarrée éventuellement par des pressions directes des candidats sur les membres plasticiens. La candidature à l'Institut implique donc l'obligation de se plier au rituel des visites, de mettre en oeuvre souvent une véritable campagne, d'élaborer une stratégie de mobilisation des relations sociales. Madame de Saint-Marceaux dénonce à plusieurs reprises toutes ces démarches dans son journal inédit: "il y a dans ces visites à faire une cuisine diplomatique qui soulève le coeur. L'honneur d'être en habit vert vaut-il tous les mensonges qu'il faut faire à des artistes sans talent". "Les tripotages de l'Académie ne sont pas croyables. Il faut avoir vu cela de près pour le croire. C'est une institution surannée et bonne à détruire".

L'examen des processus d'élection appelle quelques remarques. Le prix de Rome apparaît être une qualité nécessaire mais non suffisante à une possible élection. Le système n'est en effet pas dénué de quelques failles: Widor, Fauré, Delibes et Saint-Saëns ne sont pas lauréats du prix de Rome. Les trois premiers n'ont pas été formés au Conservatoire. Quant à Dukas et Bruneau, ils ne sont que seconds prix de Rome. Rares sont les candidats élus à leur première candidature. C 'est le cas de Lenepveu, "sans aucun talent; mauvais professeur au Conservatoire; mais romain" 10 (de notes) qui devance Fauré dont c'est alors la deuxième candidature - son eléction ne fut cependant pas si facile, puisqu'elle nécessita huit tours de scrutin: Lenepveu était classé dernier par sa section et Victorin de Joncières, classé premier, le mit en mauvaise posture, rendant la lutte serrée. C'est aussi le cas de Gustave Charpentier qui 
ne fait pas spontanément acte de candidature, mais qui répond aux pressions des membres non musiciens de l'Académie, la section de composition musicale ne l'ayant même pas sélectionné. L'âge constitue également un facteur de choix: un candidat se voit souvent devancé à sa première candidature par un autre qui en est à sa deuxième candidature et qui est plus âgé. Messager, Pierné et Bachelet attendent respectivement 73 ans, 61 ans et 65 ans pour être finalement élus à leur quatrième candidature. Il est évident que de lâge de l'élection résultent des possibilités d'influences bien différentes. Guiraud ou Dukas ne furent élus qu'un an avant leur mort. En revanche, Ambroise Thomas siège à l'Académie durant 45 ans, Saint-Saëns durant 40 ans et Théodore Dubois durant 30 ans. Le clan des organistes constitue sans doute également une "mafia" importante, les filiations se dessinant assez nettement. Ainsi Saint-Saëns apporte un soutien solide à l'election de Fauré. La "solidarité" de maître à élève apparaît aussi comme un facteur d'influence: Massenet et Dubois sont élèves d'Ambroise Thomas, Fauré de Saint-Saëns. Pour le maître, soutenir l'élève ne constitue pas obligatoirement un acte gratuit: l'élève susceptible de faire carrière peut devenir un allié éventuel. Quand un compositeur disparaît, son fauteuil peut également être attribué préférentiellement à l'un de ses élèves. Ainsi Charpentier succède-t-il à Massenet dont il était l'elève chéri. Il n'est enfin pas négligeable pour les compositeurs membres de l'Académie de compter dans leurs rangs des musiciens qui occupent des fonctions de chef d'orchestre, et qui pourraient donc favoriser l'exécution des leurs oeuvres.

La IIIème République dure 70 ans et l'on observe quelques différences entre les compositeurs officiels du début de cette longue période et ceux de la fin. L'évolution des mentalités, des modes de jugement, des critères de seléction influe sans doute sur la definition, le comportement et les attributions du compositeur officiel. Il semble que les réactions à l'Académisme soient plus ouvertes et plus violentes après la première guerre mondiale et notamment dans les années 1920. Peut-être le publics joue-t-il aussi un rôle dans cette évolution? Déjà au cours de l'immédiat avant-guerre, les Ballets Russes drainent en partie le même public que celui de l'Opéra. C'est l'occasion pour ce public de découvrir une marginalité qui n'avait pas jusque-là de scène publique à sa disposition. Les années vingt vont développer encore les possibilités d'expression de certains artistes qui vont pouvoir se faire connaître et sortir ainsi de leur anonymat. Aucun compositeur du célèbre Groupe des Six ne fut lauréat du Prix de Rome. Alors que leurs carrières se développaient, les compositeurs membres de l'Académie étaient Henri Rabaud, Georges Hüe, Alfred Bruneau... recrutés selon mêmes critères mais sans doute relégués dans leur rôle académique et pédagogique. S'ils continuent à régner sur l'enseignement et le traditionnel théâtre lyrique, les musiciens académiciens vont désormais se trouver concurrencés par la marginalité, la dissidence, les "refusés" qui ont appris à s'organiser et à se faire reconnaître.

Pour compléter cette étude, il faudra encore s'interroger sur tous les candidats malchanceux à l'Académie. Beaucoup semblent en effet posséder 
toutes les qualités requises: professeurs d'harmonie au Conservatoire, lauréats du Prix de Rome etc... Une fois étudiés dans le détail les rouages administratifs de ce pouvoir, il est bien évident qu'il conviendra d'en analyser également l'expression esthétique. Doit-on attribuer aux musiciens membres de l'Académie des Beaux-Arts une responsabilité dans l'orientation des genres et des styles? Ce n'est qu'en répondant à ces questions qu'il sera possible de dénoncer l'existence d'une censure officielle expliquant une volonté délibérée de dicter une esthétique.

Myriam Chiménes - Chargée de recherches au C.N.R.S.

\section{NOTAS}

(1) Robert M. ISHERWOOD. Music in the service of the King. France in the seventeenth Century, Ithaca. London, Comell University Press, 1973, 422 p.

(2) Myriam CHIMENES, "Le budget de la musique sous la IIIème République"; in La Musique: du théorique au politique, sous la direction de Hugues Dufourt et de Joël-Marie Fauquet. Paris, Aux amateurs de livres, 1990, p. $261-311$

(3) François FURET, La Révolution de Turgot à Jules Ferry 1770-1880, Paris, Hachette, 1988, 525 p.

(4) Pierre VAISSE, La troisième République et les peintres, recherches sur les rapports des pouvoirs publics et de la peinture en France de 1870 à 1914, Thèse de Doctorat, sous la direction de B. Dorival, Paris IV, 1980.

(5) Altred Bruneau, Musiques de Russie et musiciens de France, Paris, Librairie Charpentier. Fasquelle, 1903, p. 158.

(6) Bruno FOUCART, "La tradition ct linnovation picturale au XIXème siècle" in La tradition et linnovation dans l'art français par les peintres des salons, catalogue de lexposition au Musée Municipal de Fukuoka, mars-mai 1989, p.15.

(7) cf Tableau p. 13

(8) cf Sophie BRES, "Le scandale Ravel de 1905 " in Revue Internationale de Musique Française, $n^{\circ} 14$, juin 1984.

(9) Romain ROLLAND, Musiciens daujourd hui, Paris, Hachette, 1908, p. 223

(10) Madame de Saint-Marceaux, Joumal (inédit) 


\section{Institut de France Academie des Beaux-Arts \\ Section V: Composition Musicale (1870 - 1940)}

Fauteuil no 1:

Charles Gounod

Théodore Dubois

Gabriel Pierne

Henri Busser

Fauteuil no 2:

Victor Massé

Léo Delibes

Ernest Guiraud

Emile Paladilhe

André Messager

Alfred Bachelet

Fauteuil no 3:

Ambroise Thomas

Charles Lenepveu

C - M. Widor

Henri Rabaud

Faute uil $\mathbf{n}^{\circ}$ 4:

Félicien David

Ernest Reyer

Gabriel Fauré

Alfred Bruneau

Paul Dukas

Florent Schmitt

Fauteuil $n^{\circ}$ 5:

Henri Reber

Saint-Saëns

Georges Hüe

Fauteuil $\mathbf{n}^{\circ}$ 6:

Michel Carafa

François Bazin

Jules Massenet

Gustave Charpentier
(1818 - 1893) elu en 1866

(1837 - 1924) élu en 1894

(1863 - 1937) élu en 1925

(1872 - 1973) élu en 1938

(1822 - 1884) élu en 1872

(1836 - 1891) élu en 1884

(1837 - 1892) élu en 1891

(1844 - 1926) élu en 1892

(1853 - 1929) élu en 1926

(1864 - 1944) élu en 1929

(1811 - 1896) élu en 1851

(1840 - 1910) élu en 1896

(1844 - 1937) élu en 1910

(1873 - 1949) élu en 1918

(1810 - 1876) élu en 1869

(1823 - 1903) élu en 1876

(1845 - 1924) élu en 1909

(1857 - 1934) élu en 1925

(1865 - 1935) élu en 1934

(1870 - 1958) élu en 1936

(1807 - 1880) élu en 1853

(1835 - 1921) élu en 1881

(1858-1948) élu en 1922

(1787 - 1872) élu en 1837

$(1816-1878)$ élu en 1873

(1842 - 1912) élu en 1878

(1860 - 1956) élu en 1912
Prix Rome 1839

Prix de Rome 1861

Prix de Rome 1882

Prix de Rome 1893

Prix de Rome 1844

Prix de Rome 1859

Prix de Rome 1860

Prix de Rome 1890

Prix de Rome 1832

Prix de Rome 1865

Prix de Rome 1894

Prix de Rome 1858

Prix de Rome 1900

Prix de Rome 1879

Prix de Rome 1840

Prix de rome 1863

Prix de Rome 1887 